\title{
MEASUREMENTS OF A GAMMA-RAY BURST ABOVE 1 MeV
}

\author{
R. KOGA, G. M. SIMNETT*, and R.S. WHITE \\ Physics Dept. and Institute of Geophysics and Planetary Physics, \\ University of California, Riverside, Calif., U.S.A.
}

Summary. Observations of a burst of gamma radiation, starting at 201247 UT, 1972, May 14 are reported. The measurements were made with a $0.5 \mathrm{~m}^{2}$ actively shielded scintillator which was the front element of a double Compton telescope, during a balloon flight at an altitude of $5 \mathrm{mb}$ from Palestine, Texas. The maximum intensity of the burst was $0.10 \pm 0.02 \mathrm{~cm}^{-2} \mathrm{~s}^{-1}$ above $1 \mathrm{MeV}$, and the duration was $3.5 \pm 0.4 \mathrm{~min}$. The burst intensity during the first $2 \mathrm{~min}$ is constant to within $10 \%$. No known electronic or detector malfunction could have produced this effect, and we believe it is a real event.

The origin of the event is still unexplained. The part of the detector used for this study has virtually an omnidirectional response. A search has been made for correlative evidence, but none has been found that is conclusive. The onsets of two subflares, at S13 E78 and N12, E08, occurred one minute after the rise in the gamma-ray data. A short lived soft X-ray burst was reported from Solrad 93 min after the gamma-ray burst. It cannot be ruled out that such associations are a chance coincidence. However, simultaneous subflares from separate plage regions are reported only every few days during this time period, so the probability of a chance coincidence is less than 0.001 .

One possible explanation is that the simultaneous flares are caused by precipitating energetic protons and electrons from the high corona, with the bulk of the energy residing in the protons. Collisional bremsstrahlung from the electrons, leading to the gamma rays, might occur before the bulk of the protons lose their energy. In this way the delay in the $\mathrm{H} \alpha$ flares might be explained, but the delay in the soft X-ray emission is still puzzling.

We conclude that the origin of the gamma-ray burst is still unknown.

* Present adress : Department of Space Research, University of Birmingham, England. 